\section{As novas tecnologias de autocuidado e os riscos do autodiagnóstico pela Internet}

\author{
Paulo Roberto Vasconcellos-Silva ${ }^{1}$ \\ e Luis David Castiel²
}

Como citar: Vasconcellos-Silva PR, Castiel LD. As novas tecnologias de autocuidado e os riscos do autodiagnóstico pela Internet. Rev Panam Salud Publica. 2009;26(2):172-5.

Palavras-chave: Internet; autocuidado; acesso à informação; satisfação do consumidor; informação de saúde ao consumidor.

\footnotetext{
Universidade Federal do Estado do Rio de Janeiro, Escola de Medicina e Cirurgia. Correspondência: Rua das Laranjeiras 91/1202, CEP22240-000, Rio de Janeiro, RJ, Brasil. Fone: +55-21-2556.8804; e-mail: bioeticaunirio@yahoo.com.br

2 Fundação Oswaldo Cruz, Escola Nacional de Saúde Pública (ENSP), Departamento de Epidemiologia, Rio de Janeiro (RJ), Brasil.
}

A Internet tem se apresentado como recurso valioso no acesso às informações necessárias ao autocuidado, assim como na interação entre profissionais e seus clientes (1-3). Encorajadas por um aparato comercial de crescente influência nessa área de produção e por teóricos entusiastas (4), as tecnologias de informação e comunicação em saúde (TICS) buscam alcançar a qualidade da informação e se aprimoram quanto ao empoderamento do consumidor $(1,5)$. O contexto da elevação exponencial dos custos assistenciais, associado ao acesso hierarquizado, não-universal, aos serviços de saúde (sobretudo no cenário norte-americano) parece ter gerado demandas em saúde mal contempladas em uma sociedade cada vez mais conectada à "grande rede de informação" (4). Perante tais perspectivas, surgiram os primeiros sites de saúde direcionados à população leiga. A disponibilização de informações técnicas na Internet prenunciou o surgimento de uma nova saúde pública (6), centrada no autoesclarecimento e na autorresponsabilização dos usuários em questões ligadas à sua saúde (7). Entretanto, a disseminação de sites de duvidosa qualificação, a oferecer informações enviesadas para a divulgação de produtos comerciais, levantou questionamentos sobre a qualidade educacional das informações disseminadas nesses formatos (8).

Sillence et al. (4) identificaram novas perspectivas acerca da evolução das demandas dos consumidores em saúde nos últimos anos. Conforme esses autores, a predileção pela opinião de outros usuários que padeciam dos mesmos males deu lugar à valorização das informações qualificadas por especialistas (desde que de fácil compreensão). Embora se saiba que as informações do tipo "pergunte ao doutor", disseminadas via mensagens de e-mail sem identificação, tenham demonstrado um largo poder de alcance e sejam eficientes como complementação das formas clássicas de assistência (3), elas ainda se mostram limitadas quanto ao poder de identificar doenças à distância. Invariavelmente, as mensagens estimulavam os usuários a procurar seus médicos para maiores esclarecimentos sobre sintomas mal identificados.

Entretanto, na última década, face ao crescimento exponencial da Internet nos ambientes domésticos e profissionais, as demandas por recursos de autodiagnóstico se intensificaram na mesma medida que o clamor pela qualidade das informações pela via da certificação de sites e portais (8). Tais necessidades parecem ter sido parcialmente contempladas graças à sofisticação de algoritmos especializados no reconhecimento de doenças. A julgar pelas publicações mais recentes, os autotestes de aferição de riscos (9) parecem se adiantar como tendência promissora. Em 
suma, após a primeira onda das TICS, no formato de portais de informação, nos quais a informação é "consumida" como em um supermercado, surgem novas estratégias a equipar o consumidor em saúde em seus projetos de autocuidado. Após a era da simples, livre e descompromissada consulta de informações em portais, os apologistas da nova saúde pública anunciam a auto-administração da saúde por intermédio de tecnologias que unem a conveniência ao anonimato. As vantagens alegadas são numerosas: informação adaptada às demandas, independência do consumidor em relação à opinião de um médico, informações não comprometidas por razões comerciais e sigilo e anonimato assegurados, entre outras vantagens evidentes.

\section{Os sites de autodiagnóstico e os seus problemas}

Nos últimos anos, impulsionados pelas tecnologias emergentes de software e algoritmos para troca de dados (além da acentuada aceleração dos fluxos pela banda larga da Internet), surgiram diversos novos portais especializados no diagnóstico segundo descrições de sintomas. Entre vários aplicativos de autodiagnóstico que se apresentam à busca per mecanismos como o google.com, destacam-se o "familiydoctor.org", o site comercial "eCureme.com" e o portal do sistema de saúde inglês (tabela 1).

Uma consulta pelo item "dor de cabeça" (headache) no familiydoctor.org guia o usuário a uma variada lista de diagnósticos, encabeçada por gripe, meningite, hematoma subdural e acidente vascular encefálico. $\mathrm{O}$ sistema de diagnósticos online do site NHS Direct apresenta resultados mais enfáticos. Dois cliques direcionam o usuário às "dores de cabeça de adultos". Logo em seguida, o algoritmo pergunta se essas dores são persistentes, de instalação súbita, se têm piorado e atrapalhado nas ocupações cotidianas (http://www. nhsdirect.nhs.uk/help/bodykey/Questions/Index. aspx?nodes $=\% 2 b O e K I t a h 40 Q \% 3 d)$. Para os casos positivos há um alerta categórico, com número de tele- fone para requisição imediata de uma ambulância e remoção para um serviço de emergência.

Embora a alta tecnologia tenha sido desenvolvida para garantir a ágil recuperação de informações, algumas dificuldades parecem tipicamente refratárias aos esforços dos especialistas, conforme descrevem Nijland et al. (10). Esses autores avaliaram, utilizando abordagem qualitativa, a opinião de usuários holandeses acerca dos serviços oferecidos pelos sites Medicinfo, Praktijkinfo e Dokterdokter (tabela 1) - todos certificados nos padrões ISO 9000:2000. Os usuários são cadastrados com senhas e, com isso, obtêm livre acesso a glossários de termos médicos e doenças, além de aplicativos de autodiagnóstico. Entre esses aplicativos, o Symptom Scan consiste em um autoteste útil para identificar causas e sintomas e disponibiliza uma lista de probabilidades de diagnósticos para cada tipo de dor ou desconforto. Os sintomas são localizados pelo cursor em um "corpo virtual" e, com base em questionários para descrição de indícios clínicos de doenças específicas, chega-se aos diagnósticos e, se pertinente, ao aconselhamento padronizado (em casos que não sejam de urgência). Decerto tal praticidade economizaria recursos financeiros e tempo, com a confidencialidade assegurada, a não ser pelo fato de que muitos usuários demonstram dificuldades em localizar no corpo virtual sintomas como cansaço, tonteiras e insônia. Da mesma forma, sintomas que se apresentam simultaneamente em vários segmentos, como dores musculares generalizadas e cãibras, também causaram dúvidas, o que tendia a incitar o retorno às buscas pelo Google. Nijland et al. (10) mostram ainda que os problemas encontrados mais frequentemente se concentraram na "inadequação das informações às realidades dos usuários", nos desníveis entre o léxico técnico e o leigo e nos recursos de navegação pouco intuitivos ou ineficientes.

Afora os problemas ligados às tecnicidades do desenho das páginas e às questões de acessibilidade aos conteúdos mais relevantes, parece haver proble-

TABELA 1. Ferramentas para autodiagnóstico em 14 sites da Internet

\begin{tabular}{ll}
\hline \multicolumn{1}{c}{ Nome } & \multicolumn{1}{c}{ Endereço } \\
\hline Familiydoctor.org & http://familydoctor.org/online/famdocen/home/tools/symptom.html \\
eCureme.com & http://www.ecureme.com/emyhealth/symptoms/fm_front.asp \\
Revolution Health & http://www.revolutionhealth.com/symptom-checker/ asp \\
Health Central & http://www.healthcentral.com/symptom-checker/ \\
Easy Diagnosis & http://easydiagnosis.com/modules.html \\
Medicine Net & http://www.medicinenet.com/symptoms_and_signs/article.htm \\
NHS Direct & http://www.nhsdirect.nhs.uk/help/bodykey \\
Medicinfo ${ }^{b}$ & http://www.medicinfo.nl/virtueellichaam \\
Praktijkinfo & http://www.praktijkinfo.nl/info/huisarts.asp?actief=hoofdmenu \\
Your Diagnosis & http://www.yourdiagnosis.com// \\
Self Diagnosis & http://www.selfdiagnosis.co.uk/ \\
ABC Homeopathy & http://abchomeopathy.com/self.htm \\
Dokterdokter & http://www.dokterdokter.nl \\
Interactive Health Symptom Checker & http://health.msn.com/symptoms/default.aspx \\
\hline a Sites acessados em julho de 2009. & \\
b Conforme Nijland et al. (10). &
\end{tabular}


mas que apontam para raízes mais profundas, ligadas à forma de entender o processo de autocuidado frente às múltiplas demandas da clientela. No site Medicinfo, a descrição de uma simples dor de cabeça pode resultar na estimativa de $96 \%$ de chances de enxaqueca, $1,1 \%$ de tumor cerebral e $0,1 \%$ de meningite. Uma resposta em formato probabilístico pode ser considerada satisfatória pelos desenvolvedores do site, porém afronta os dados obtidos por outros autores que dão conta da manifesta aversão dos pacientes às respostas no formato de estatísticas (11). Em geral, segundo os depoimentos reproduzidos por Nijland et al. (10), os pacientes se mostram reticentes na interpretação de informações apresentadas nesses formatos - perante uma dor de cabeça persistente, como encarar a probabilidade de 1,1\% de tumor cerebral, mesmo que muito menor do que a probabilidade de uma enxaqueca? Esse tipo de informação, assim como as indeterminações de qualquer ordem, parece incitar os protopacientes à procura urgente de especialistas, o que contradiz a ideia original da autoadministração de saúde.

Esses mesmos autores (10) também observam que a maior parte das informações foi produzida para dar conta de situações de doença estabelecida, e não dos frequentes e fugazes desconfortos que nos afligem no cotidiano. Foram também descritas muitas dificuldades na localização de informações consideradas pertinentes entre os excessos disponibilizados pelo aplicativo-glossário. Oscilando entre aconselhamentos elementares (que beiravam as obviedades do senso comum) e a extrema complexidade técnica de alguns termos, os usuários holandeses concluíam ironicamente pela necessidade de um "prediagnóstico" ou, pelo menos, de maior qualificação técnica que os habilitasse às consultas. Em síntese, o paradoxo: se um dos objetivos do autocuidado era a libertação dos pacientes da tutela de médicos paternalistas, passou a existir a necessidade de capacitação para o exercício dessas práticas libertárias, que aparentemente não podem ser utilizadas por qualquer um para obter informações sobre qualquer tipo de tema.

\section{O papel das tecnologias nas interlocuções}

Os trabalhos que situam as perspectivas dos usuários acerca de sistemas de TICS parecem bem mais escassos do que aqueles que advogam as inovações tecnológicas (por vezes como fins em si) $(1,12)$. No que concerne ao segmento humano do paradigma linear da comunicação (13), parece haver uma escassez de pesquisas de recepção acerca dos sentidos e significados que circundam a ideia de autossuporte. Há estudos que apontam para divergências entre a hierarquização técnica e leiga de sintomas considerados graves ou prosaicos - pacientes tendem a desconsiderar a tosse, por exemplo, como sintoma digno de maiores investigações (o oposto ocorre com as queixas de cansaço). Em ambos os casos, observa-se o mesmo resultado - desconfianças e incertezas que induzem à busca de especialistas (10).
O que parece corroborar os anseios por interlocução e formação de parcerias foi a predileção pelas redes de comunicação online com profissionais e cuidadores ou as mediadas por correio eletrônico (1-3, 5, 10). A principal vantagem apontada foi, em síntese, o acesso conveniente a fontes de consulta com informações altamente personalizadas. A triagem digital pelo autodiagnóstico parece haver frustrado expectativas, visto que os usuários tendem a categorizá-la como um tipo de recurso centrado em tecnologia biomédica anteposta aos seus problemas reais cotidianos (10). As tecnologias adialógicas (como a do autodiagnóstico) têm proliferado como formas eficientes de intervir sobre os processos de saúde-doença-cuidado, o que pode vir, paradoxalmente, a disseminar temores, ansiedades e consultas desnecessárias.

Talvez as referidas falhas se devam à concepção da eHealth (14) como um objeto de intervenções tecnológicas unilaterais, centradas meramente na disponibilização automática de conteúdos técnicos. Como resultado, obtêm-se a clássica disjunção semântica (e de léxicos) (15) por meio de algoritmos que deveriam abreviar dificuldades ao invés de ampliar necessidades na direção de novos recursos. Na maré vazante dos primeiros clamores por sites e informações qualificadas, não são poucos, nem inexpressivos, os autores que discutem formas de inverter a polaridade do paradigma linear, situando os usuários da eHealth como legítimos protagonistas no processo de autocuidado $(1,2,5,12$, 16-20). O lugar vacante das tecnologias para interlocuções deixa-se entrever nos estudos que dão conta das demandas por redes solidárias e grupos de apoio que habilitem à autonomia das livres decisões fundamentadas no diálogo com profissionais que adotem tais premissas (5). O desafio da comunicação, no seu senso mais amplo, tem-nos proposto o encontro com a alteridade em novos formatos de assistência (15), centrados no protagonismo de pacientes e nas partilhas intersubjetivas para estabelecimento de parcerias perenes.

\section{CONCLUSÕES}

Há fontes que dão conta de uma certa forma de nostalgia pelo conservadorismo dos pacientes de outrora. Ao final dos anos 1970, apenas 5\% dos pacientes mudavam de médico por se sentirem mal assistidos e apenas um em 10 pensava em mudar de profissional (21). No início do terceiro milênio, observa-se uma inclinação dos consumidores em saúde à solidão em suas buscas por aconselhamento impessoal e distanciado, a julgar pelas tendências aqui descritas. Após os primeiros esforços no sentido de conferir qualidade e isenção aos conteúdos sobre saúde de sites dirigidos à população leiga, os usuários parecem se perder em meio a aconselhamentos uniformemente pífios ou alarmistas. Apesar de a oferta de mensagens merecer grandes investimentos, pouco se estuda sobre como os usuários interpretam as informações disponibilizadas no que concerne ao que lhes é substantivo no cuidar de si. O paradigma linear da comunicação parece instruir 
a unidimensionalidade que permeia a maioria dos estudos na área de adventos tecnológicos. Recorrentemente, as perspectivas nesse campo se concentram na relação homem-máquina, com desfechos concentrados no aprimoramento da máquina a fim de se alcançar o alinhamento comportamental do ser humano. As mídias de massa revigoram cotidianamente um conceito diretamente ligado à lógica da autorresponsabilização em saúde - o empoderamento individual - na crença de que o somatório de nossas saúdes individuais se prestaria à promoção de uma sociedade sadia. Não obstante, pacientes e cuidadores não perceberam suas expectativas como atendidas frente aos novos recursos interativos que, paradoxalmente, parecem incitar ao círculo vicioso da busca pelos especialistas. Por outro lado, os recursos tecnológicos poderiam estar centrados na simplicidade das conversações, ampliando os caminhos para a universalidade ao estimular, sob variados pretextos, a formação de redes de profissionais, pacientes e cuidadores. Sob o imperativo da interlocução, as demandas peculiares tenderiam a se aglutinar em sub-redes, o que também pode oferecer promissores espaços para a integralidade.

Agradecimentos. Esta pesquisa foi financiada pelo Conselho Nacional de Pesquisa e Desenvolvimento Científico e Tecnológico - MCT/CNPq (edital No. 014/2008 - Universal).

\section{SYNOPSIS}

\section{New self-care technologies and the risk of self-diagnosis through the Internet}

Encouraged by a solid commercial structure and growing cultural influence, information and communication technologies for health (ICTH) appear as a natural reference point for matters of self-care. As a first trend, ICTH involved end user consultations to websites specializing in health. Soon after, the development of algorithms focused on the identification of illnesses to meet the needs of consumers seeking distant, impersonal technical advice. Despite the comfort and confidentiality, there is evidence that such resources have generated more doubt and anxiety than meaningful clarifications. According to the literature, users seem to have difficulties in identifying and naming their own symptoms, in addition to having to choose between shallow or alarmist advice. Despite the investments made in providing information, most sources of information do not rely on previous cultural studies about how users interpret their health conditions when resorting to selfdiagnosis, which ends up by pushing them, paradoxically, to look for specialists.

Key words: Internet; self-care; access to information; consumer satisfaction; consumer health information.

\section{REFERÊNCIAS}

1. Kerr C, Murray E, Stevenson F, Gore C, Nazareth I. Internet interventions for long-term conditions: patient and caregiver quality criteria. J Med Internet Res. 2006;8(3):e13.

2. Tjora A, Tran T, Faxvaag A. Privacy vs. usability: a qualitative exploration of patients' experiences with secure Internet communication with their general practitioner. J Med Internet Res. 2005;7(2):e15.

3. Umefjord G, Hamberg K, Malker H, Petersson G. The use of an Internet-based Ask the Doctor Service involving family physicians: evaluation by a web survey. Fam Pract. 2006;23(2):159-66.

4. Sillence E, Briggs P, Harris P, Fishwick L. Going online for health advice: changes in usage and trust practices over the last five years. Interact Comput. 2007;19(3): 397-406.

5. Verhoeven F, van Gemert-Pijnen L, Dijkstra K, Nijland N, Seydel E, Steehouder $\mathrm{M}$. The contribution of teleconsultation and videoconferencing to diabetes care: a systematic literature review. J Med Internet Res. 2007;9(5):e37.

6. McLeod SD. The quality of medical information on the Internet. A new public health concern. Arch Ophthalmol. 1998; 116(12):1663-5.

7. Eysenbach G, Köhler C, Yihune G, Lampe $\mathrm{K}$, Cross P, Brickley D. A framework for improving the quality of health informa- tion on the world-wide-web and bettering public e-health: the MedCERTAIN approach. Stud Health Technol Inform. 2001;84(2):1450-4.

8. Castiel LD, Vasconcellos-Silva PR. Internet e o autocuidado em saúde: como juntar os trapinhos? Hist Cienc Saude Manguinhos. 2002;9(2):291-314.

9. Chambers R. Supporting self-care. Em: Chambers R, Wakley G, Blenkinsopp A. Supporting self care in primary care. Oxford: Oxford Press; 2006. Pp. 3-13.

10. Nijland N, van Gemert-Pijnen J, Boer $\mathrm{H}$, Steehouder MF, Seydel ER. Evaluation of Internet-based technology for supporting self-care: problems encountered by patients and caregivers when using self-care applications. J Med Internet Res. 2008;10(2):e13.

11. Tamburini M, Gangeri L, Brunelli C, Boeri P, Borreani C, Bosisio M et al. Cancer patients' needs during hospitalisation: a quantitative and qualitative study. BMC Cancer. 2003;3:e12.

12. Hesse BW, Shneiderman B. eHealth research from the user's perspective. Am J Prev Med. 2007;32(5 Suppl):97-103.

13. Sfez L. Crítica da comunicação. São Paulo: Loyola; 1994:41.

14. Eisenbach G. What is eHealth? J Med Internet Res. 2001;3(2):e20.

15. Vasconcellos-Silva PR, Rivera FJU, Siebeneichler FB. Healthcare organizations, linguistic communities, and the emblematic model of palliative care. Cad Saude Publica. 2007;23(7):1529-38.

16. Gustafson DH, Wyatt JC. Evaluation of eHealth systems and services. BMJ. 2004; 328(7449):1150.

17. Institute of Medicine. Crossing the quality chasm: a new health system for the 21st century. Washington DC: National Academies Press; 2001.

18. Katz SJ, Moyer CA. The emerging role of online communication between patients and their providers. J Gen Intern Med. 2004;19(9):978-83.

19. Patt MR, Houston TK, Jenckes MW, Sands DZ, Ford DE. Doctors who are using e-mail with their patients: a qualitative exploration. J Med Internet Res. 2003;5(2):e9.

20. Hobbs J, Wald J, Jagannath YS, Kittler A, Pizziferri L, Volk LA, et al. Opportunities to enhance patient and physician e-mail contact. Int J Med Inform. 2003;70(1):1-9.

21. Ritchie J, Jacoby A, Bone M. Access to primary health care: an enquiry carried out on behalf of the United Kingdom Health Departments. London: HMSO; 1981.

Manuscrito recebido em 27 de agosto de 2008. Aceito em 6 de dezembro de 2008. 